\title{
Fermion dipole moment and holography
}

\author{
Manuela Kulaxizi ${ }^{a, b}$ and Rakibur Rahman ${ }^{c}$ \\ ${ }^{a}$ School of Mathematics, Trinity College Dublin, \\ Dublin 2, Ireland \\ ${ }^{b}$ Lorentz Institute for Theoretical Physics, Leiden University, \\ P.O. Box 9506, Leiden 2300RA, The Netherlands \\ ${ }^{c}$ Max-Planck-Institut für Gravitationsphysik (Albert-Einstein-Institut), \\ Am Mühlenberg 1, D-14476 Potsdam-Golm, Germany \\ E-mail: manuela.kulaxizi@gmail.com, rakibur.rahman@ulb.ac.be
}

ABSTRACT: In the background of a charged AdS black hole, we consider a Dirac particle endowed with an arbitrary magnetic dipole moment. For non-zero charge and dipole coupling of the bulk fermion, we find that the dual boundary theory can be plagued with superluminal modes. Requiring consistency of the dual CFT amounts to constraining the strength of the dipole coupling by an upper bound. We briefly discuss the implications of our results for the physics of holographic non-Fermi liquids.

KEYWORDS: Gauge-gravity correspondence, Holography and condensed matter physics (AdS/CMT)

ARXIV EPRINT: 1507.08284 


\section{Contents}

1 Introduction 1

2 Fermion dipole coupling \& holographic diagnosis 2

2.1 Fermion fluctuations 3

2.2 Causality violation \& constraints 5

$\begin{array}{lll}2.3 & \text { WKB approximation: a numerical study } & 7\end{array}$

3 Implications \& remarks $\quad 9$

\section{Introduction}

Gauge/gravity duality [1-3] may serve as a diagnostic tool for assessing the consistency of a theory since it allows one to explore otherwise unaccessible regions of the parameter space. Some pathological features of the theory may become more manifest in one side of the duality or the other. Notable examples include the observed violation of causality in the boundary CFT for some apparently healthy bulk duals with generic couplings: ghost-free Gauss-Bonnet gravity in five dimensions [4-7], and Lovelock and other higher-derivative theories of gravity [8-14]. The bulk couplings are constrained by the consistency of the boundary theory, and in the context of field theory this can be understood as constrains imposed by unitarity [7, 15, 16]. It was not until recently [17] that these bulk gravity theories are shown to have unambiguous signs of pathologies at the classical level.

The techniques of ref. [17] however cannot be employed for electromagnetic (EM) couplings. ${ }^{1}$ To date, there is no known classical inconsistency for a massive charged spin-1 particle to have an arbitrary magnetic dipole moment $[18,19]$; only quantum consistency requirements, like perturbative renormalizability and tree level unitarity, spell out a unique bare value at the Lagrangian level [20,21]. Yet it was argued in ref. [22] that once the theory is placed in a charged AdS black hole background, a generic dipole coupling would afflict the dual CFT with superluminal modes. A similar story is expected for the magnetic dipole coupling of a massive Dirac fermion, which we are going to consider in this article.

Note that if the EM interactions preserve Lorentz, parity and time-reversal symmetries, a spin- $\frac{1}{2}$ particle of mass $m$ may possess a charge $q$ and a magnetic dipole moment $\mu_{m}$. The Lagrangian for a Dirac field $\Psi$ incorporates them through the minimal coupling and the Pauli term:

$$
\mathcal{L}=-i \bar{\Psi}\left(\gamma^{\mu} D_{\mu}-m\right) \Psi+q \lambda \bar{\Psi} \gamma^{\mu \nu} F_{\mu \nu} \Psi
$$

\footnotetext{
${ }^{1}$ For EM couplings, the scattering amplitude goes like the Mandelstam variable $s$, instead of $s^{2}$. This gives zero time delay for a fast particle crossing a shock wave. We thank A. Zhiboedov for this point.
} 
where the covariant derivative is $D_{\mu}=\partial_{\mu}-i q A_{\mu}$, and the $\Gamma$ matrices are the same as those of ref. [23]. The magnetic dipole moment has the value

$$
\mu_{m}=\frac{q}{2 m}(2+4 m \lambda) .
$$

The minimal coupling alone gives a gyromagnetic ratio of 2, as shown by Dirac. But nothing forbids a non-zero contribution from the dipole coupling since the classical electrodynamics is consistent for an arbitrary value of $\lambda$. However, the Pauli term renders the theory powercounting non-renormalizable, and so quantum consistency requires that $\lambda$ vanish at the tree level [24]. In an effective field theory, though, quantum corrections would generate a small but non-zero value for this coupling constant.

When coupled to gravity as well, the system would be described by the Lagrangian:

$$
\mathcal{L}=-i \sqrt{-\operatorname{det} g}\left[\bar{\Psi}\left(\Gamma^{\mu} \nabla_{\mu}-m\right) \Psi+i q \lambda \bar{\Psi} \Gamma^{\mu \nu} F_{\mu \nu} \Psi\right]
$$

where $\Gamma^{\mu}=e_{a}^{\mu} \gamma^{a}$ is given by the flat-space $\gamma$-matrices and the vierbein, the covariant derivative $\nabla_{\mu}=D_{\mu}+\frac{1}{4} \hat{\omega}_{\mu a b} \gamma^{a b}$ incorporates the spin connection, and $\Gamma^{\mu \nu}=e_{a}^{\mu} e_{b}^{\nu} \gamma^{a b}$.

When the fermion is a probe in an AdS Reissner-Nordström background, the system (1.3) has a boundary dual that captures the physics of a non-Fermi liquid [25, 26]. The dipole coupling has been extensively studied in bottom-up [23, 27-38] and top-down [39-47] AdS/CFT models. In this article we will use the same setup to show that holography poses a constraint on the bulk dipole coupling.

The organization of the article, with section 2 constituting the bulk, is as follows. Given the AdS Reissner-Nordström geometry, we consider in section 2.1 the equations of motion of a probe fermion, which simplify considerably in some suitable region of the parameter space, where the momentum, frequency, chemical potential and mass-squared are all very large. The resulting zero-energy Schrödinger problem is considered in section 2.2 to show that there exist normalizable solutions peaked near the boundary. The conditions that allow for such solutions also allow us to compute the group velocity of certain modes coupled to the boundary fermionic operator. We derive an upper bound on the strength of the dipole coupling, which when violated plagues the boundary theory with acausality. This result is reconfirmed in section 2.3, where we use the WKB method and compute numerically the group velocity of the boundary modes from the resulting Bohr-Sommerfeld quantization condition. In section 3, we make some remarks and briefly discuss the implications of our results for the physics of holographic non-Fermi liquids.

\section{Fermion dipole coupling \& holographic diagnosis}

The charged AdS black hole is a solution of the Einstein-Maxwell system in $\mathrm{AdS}_{d+1}$ [48-50]:

$$
d s^{2}=\frac{r^{2}}{L^{2}}\left[-f(r) d t^{2}+d \vec{x}^{2}\right]+\frac{L^{2}}{r^{2}} \frac{d r^{2}}{f(r)}, \quad A_{\sigma}=\mu\left(1-\frac{r_{0}^{d-2}}{r^{d-2}}\right) \delta_{\sigma}^{t},
$$


where $L$ is the AdS radius. The function $f(r)$ and $\mu$ generically depend on the mass and the charge of the black hole, but reduce in the zero temperature limit of the black hole to

$$
f(r)=1+\frac{d}{d-2}\left(\frac{r_{0}}{r}\right)^{2 d-2}-\frac{2(d-1)}{d-2}\left(\frac{r_{0}}{r}\right)^{d}, \quad \mu= \pm \sqrt{\frac{1}{2} d(d-1)} \frac{g_{F} r_{0}}{(d-2) L^{2}},
$$

where $r_{0}$ is the horizon radius and $g_{F}$ is the effective dimensionless gauge coupling $[25,26]$.

In this background, it is a great convenience that certain cubic and higher couplings could be investigated at the level of a quadratic Lagrangian of the bulk field(s). From the CFT point of view, it means that 2-point functions at a finite charge density contain information about certain 3- and higher-point functions.

\subsection{Fermion fluctuations}

Considering a probe Dirac fermion in the $(d+1)$-dimensional AdS Reissner-Nordström geometry, given by eqs. (2.1) and (2.2), allows us to study the dynamics of a dual fermionic operator $\mathcal{O}$ at zero temperature but at a finite chemical potential $\mu$. The mass $m$ of the field, taken to be positive without any loss of generality, is related to the conformal dimension $\Delta$ of the operator as: $\Delta=\frac{d}{2}+m L$, assuming a stable CFT for small mass [25, 26]. The $d$-dimensional CFT has a global U(1) symmetry and an associated conserved current $J_{\mu}$, which the holographic duality maps to the bulk $\mathrm{U}(1)$ gauge field. The CFT operator $\mathcal{O}$ has a charge $q$ under the global $\mathrm{U}(1)$.

In the following we restrict ourselves to $d=3$. For future convenience, let us choose the black hole charge $Q$ such that $\operatorname{sgn}(q)=\operatorname{sgn}(Q)=\operatorname{sgn}(\mu)$. Without any loss of generality, we take them all to be positive. The dynamics of a probe spin- $\frac{1}{2}$ field in the background is governed by the Dirac equation obtained by varying of the action (1.3):

$$
\left(\Gamma^{\mu} \nabla_{\mu}-m\right) \Psi+i q \lambda \Gamma^{\mu \nu} F_{\mu \nu} \Psi=0 .
$$

Rotational invariance allows us to consider small perturbations of the form $\Psi(r, t, x)$, which can be Fourier transformed as

$$
\Psi(r, t, x)=\left(-\operatorname{det} g g^{r r}\right)^{-\frac{1}{4}} \int \frac{d \omega d k}{(2 \pi)^{2}} \psi(r, \omega, k) e^{i(k x-\omega t)} .
$$

Substituting (2.4) into eq. (2.3) one finds:

$$
\left(\gamma^{r} \partial_{r}-m \sqrt{g_{r r}}\right) \psi-i\left[\sqrt{\frac{g_{r r}}{-g_{t t}}} \gamma^{t}\left(\omega+q \mu\left(1-\frac{r_{0}}{r}\right)\right)-\sqrt{\frac{g_{r r}}{g_{i i}}} \gamma^{x} k-\frac{2 q \lambda \mu r_{0}}{\sqrt{-g_{t t}} r^{2}} \gamma^{r t}\right] \psi=0 .
$$

Let us introduce a new radial variable:

$$
\zeta \equiv \frac{r_{0}}{r}, \quad 0 \leq \zeta \leq 1 .
$$

Now the boundary is at $\zeta=0$ and the horizon at $\zeta=1$. We also define the parameters:

$$
\tilde{\omega}=\frac{\omega L^{2}}{r_{0}}, \quad \tilde{k}=\frac{k L^{2}}{r_{0}}, \quad \tilde{\mu}=\frac{q \mu L^{2}}{r_{0}},
$$


Denoting a derivative w.r.t. $\zeta$ by a prime, we rewrite the Dirac equation (2.5) as

$$
\psi^{\prime}+\mathcal{A} \psi=0,
$$

where the matrix-valued function $\mathcal{A}=\mathcal{A}(\zeta)$ is given in terms of the Pauli matrices $\sigma^{1,2,3}$ :

$$
\mathcal{A}=\left(\begin{array}{cc}
\frac{-\tilde{k}+2 \tilde{\mu} \zeta \lambda / L}{\sqrt{f(\zeta)}} \sigma^{1}+\frac{\Omega(\zeta)}{f(\zeta)} i \sigma^{2}-\frac{m L}{\zeta \sqrt{f(\zeta)}} \sigma^{3} & \mathbf{0} \\
\mathbf{0} & \frac{\tilde{k}+2 \tilde{\mu} \zeta \lambda / L}{\sqrt{f(\zeta)}} \sigma^{1}+\frac{\Omega(\zeta)}{f(\zeta)} i \sigma^{2}-\frac{m L}{\zeta \sqrt{f(\zeta)}} \sigma^{3}
\end{array}\right)
$$

with

$$
f(\zeta)=1-4 \zeta^{3}+3 \zeta^{4}, \quad \Omega(\zeta)=\tilde{\omega}+\tilde{\mu}(1-\zeta) .
$$

For the sake of better physical understanding, let us apply the operator $\left(\mathbf{1} \partial_{\zeta}-\mathcal{A}\right)$ on eq. (2.8) to obtain a multi-channel coupled Schrödinger problem:

$$
-\psi^{\prime \prime}+\left(\mathcal{A}^{2}-\mathcal{A}^{\prime}\right) \psi=0
$$

Because the matrix $\mathcal{A}$ is block diagonal, the 2-component chiral spinors comprising $\psi$ decouple from each other. In other words, we can write

$$
\psi=\left(\begin{array}{c}
\psi_{-} \\
\psi_{+}
\end{array}\right)
$$

and split eq. (2.11) into two: one with $\psi_{-}$alone and the other with $\psi_{+}$. However, the components of $\psi_{ \pm}$themselves are non-trivially coupled, which makes the problem difficult.

We will now focus on some suitable region of the parameter space, where the momentum, frequency, chemical potential and mass-squared are all very large. The system (2.11) will be much simplified in this regime. To spell out the region of parameter space to be considered, let us first define some dimensionless ratios:

$$
u \equiv \frac{\tilde{\omega}}{\tilde{k}}, \quad v \equiv \frac{\tilde{\mu}}{\tilde{k}}, \quad w \equiv \frac{m^{2} L^{2}}{\tilde{k}} .
$$

Now we take the following limit of large momentum that simultaneously sets the frequency, chemical potential and mass-squared large as well:

$$
\tilde{k} \gg 1, \quad \text { with } \quad u, v, w=\text { constant. }
$$

This limits our focus at a small corner of the vast parameter space of the boundary field theory: one is choosing a large scaling dimension for the operator $\mathcal{O}$, tuning the chemical potential as large as $\Delta^{2}$, and looking at comparable frequencies and momenta.

Note that because of the large chemical potential the spinor field is subject to instability or condensation deep inside the bulk where the background EM field is sufficiently strong. These effects are negligible as long as the EM field invariant is small: $\left|F_{\mu \nu}\right|^{2} \ll \frac{m^{4}}{q^{2}}$. In the vicinity of the boundary, a region $[0, \zeta]$ does not encounter these issues as long as $\zeta$ is small enough:

$$
\left(\frac{v}{w}\right)^{2} \zeta^{4} \ll 1 .
$$


This means in particular that if $v, w \sim 1$, as is chosen in section 2.2, the idea of a longlived propagating particle makes sense only in the near-boundary region, which is where one should focus at. As long as the condition (2.15) is satisfied, other values of the parameters may render it safe to consider deep regions in the bulk as well (see section 2.3).

\subsection{Causality violation \& constraints}

Let us set the AdS radius to unity: $L=1$. In the limit (2.14), the two components of $\psi_{ \pm}$ decouple from each other, except for the near-horizon region. The single-channel quantum mechanical problem one obtains in the limit (2.14) is a zero-energy Schrödinger equation:

$$
-\frac{1}{\tilde{k}^{2}} \psi_{ \pm}^{\prime \prime}+V_{ \pm}(\zeta) \psi_{ \pm}=0
$$

where the index labeling the different components of $\psi_{ \pm}$has been omitted (since the equations are identical), and the approximate potential function is given by

$$
V_{ \pm}(\zeta)=\frac{w}{\tilde{k} \zeta^{2} f(\zeta)}+\frac{(1 \pm 2 \lambda v \zeta)^{2} f(\zeta)-(u+v-v \zeta)^{2}}{f^{2}(\zeta)}
$$

Despite a $\frac{1}{\widetilde{k}}$-dependence, the first term in $V_{ \pm}(\zeta)$ becomes increasingly important as one approaches the boundary, and makes the potential go to $+\infty$ as $\zeta \rightarrow 0$. On the other hand, deep inside the bulk $V_{ \pm}(\zeta)$ has large negative values. Therefore, if the potential has a local minimum in the vicinity of the boundary, it will also have a local maximum farther inside the bulk. Close to the boundary a pair of local minimum and maximum will always exist, either for the $\psi_{-}$or the $\psi_{+}$modes, if the parameters obey

$$
\begin{aligned}
u+v & =2|\lambda|, \\
4 \lambda^{2} & >1 .
\end{aligned}
$$

To see this, let us take the near-boundary expansion of the derivative of the potential:

$$
V_{ \pm}^{\prime}(\zeta)=-\frac{2 w}{\tilde{k} \zeta^{3}}+(u+v \pm 2 \lambda)+2 v^{2}\left(4 \lambda^{2}-1\right) \zeta-12\left(8 \lambda^{2}-1\right) \zeta^{2}+\mathcal{O}\left(\zeta^{3}\right)
$$

Depending on the sign of $\lambda$, the constant piece $(u+v \pm 2 \lambda)$ can always be set to zero either for the $\psi_{-}$or the $\psi_{+}$modes. This gives the choice of parameters (2.18). Provided the condition (2.19) is also fulfilled, $V_{ \pm}^{\prime}(\zeta)$ has a zero at the point

$$
\zeta_{\min } \approx\left[\frac{w}{\tilde{k} v^{2}\left(4 \lambda^{2}-1\right)}\right]^{\frac{1}{4}} .
$$

For $v, w \sim 1$, this is arbitrarily close to the boundary in the limit $\tilde{k} \rightarrow \infty$. That $\zeta_{\text {min }}$ is in fact a minimum of the potential can be understood from its second derivative:

$$
V_{ \pm}^{\prime \prime}\left(\zeta_{\min }\right) \approx 8 v^{2}\left(4 \lambda^{2}-1\right)>0
$$




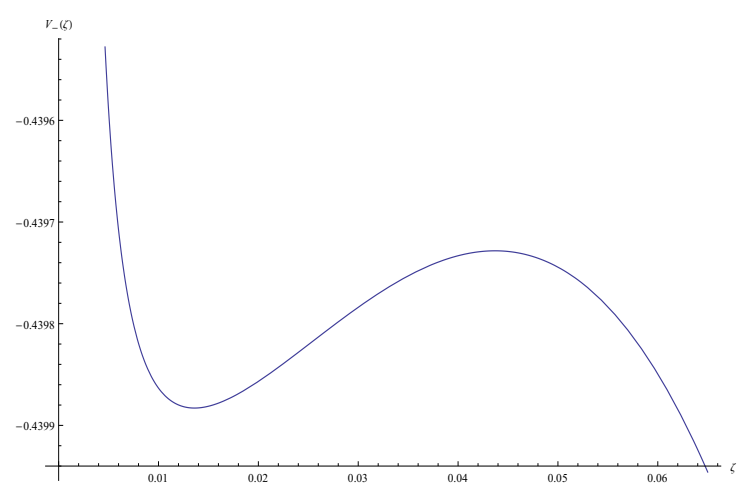

Figure 1. $V_{-}(\zeta)$ is plotted for $\tilde{k}=10^{8}, v=$ $1, w=1$ and $\lambda=0.6$, subject to the conditions (2.18)-(2.19). The potential develops a well close to the boundary. $V_{+}(\zeta)$ has an identical plot with the sign of $\lambda$ flipped.

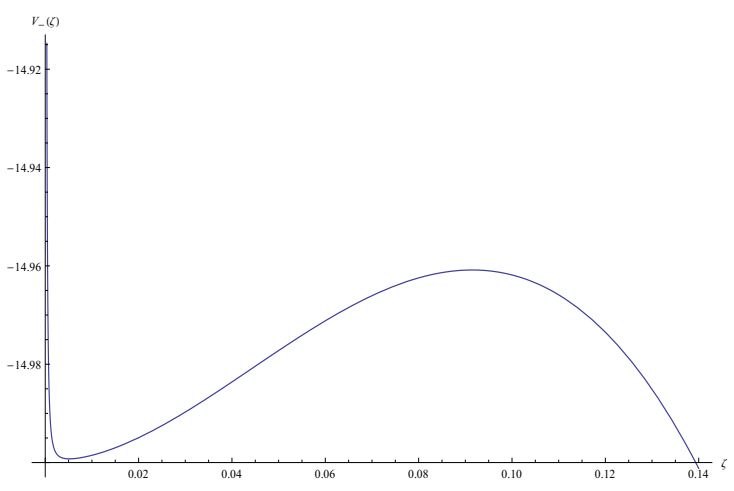

Figure 2. $V_{-}(\zeta)$ is plotted for $\tilde{k}=10^{8}, v=$ $1, w=1$ and $\lambda=2.0$, subject to the conditions (2.18)-(2.19). The potential develops a well close to the boundary. $V_{+}(\zeta)$ has an identical plot with the sign of $\lambda$ flipped.

Furthermore, the choices (2.18)-(2.19) also ensure that there is maximum "slightly" away from the boundary, where the $\frac{1}{\tilde{k}}$-term is negligible; it is located at the point

$$
\zeta_{\max } \approx \frac{v^{2}}{6}\left(\frac{4 \lambda^{2}-1}{8 \lambda^{2}-1}\right)
$$

which is at least an order of magnitude smaller than unity for $v \approx 1$.

Under the conditions (2.18)-(2.19), therefore, the potential develops a well in the vicinity of the boundary, where the EM field is sufficiently weak. Indeed, the inequality (2.15) holds very well for any $\zeta \in\left[0, \zeta_{\max }\right]$. The potential well will admit normalizable solutions of the zero-energy Schrödinger problem (2.16) that are peaked near the boundary. For $\lambda>\frac{1}{2}$, the $\psi_{-}$modes will have such solutions, whereas if $\lambda<-\frac{1}{2}$, they occur for the $\psi_{+}$modes.

The normalizable zero-energy solutions of the quantum mechanical problem correspond to the existence of (quasi)particles in the dual CFT that are coupled to the operator $\mathcal{O}$. As one expects from refs. [4, 5, 8-14, 22], the boundary theory may suffer from causality violation for a generic dipole coupling. Such an inconsistency indeed arises unless the bulk coupling parameter $\lambda$ is appropriately constrained, as we are going to see. Note that the phase velocity of the modes coupled to $\mathcal{O}$ is given by

$$
c_{p} \equiv \frac{\tilde{\omega}}{\tilde{k}}=u \text {. }
$$

When this is compared with the condition (2.18), one gets a simple dispersion relation for the boundary modes, which takes the form:

$$
c_{p}=2|\lambda|-\frac{\tilde{\mu}}{\tilde{k}} .
$$

This in turn allows one to compute the group velocity:

$$
c_{g} \equiv c_{p}+\tilde{k}\left(\frac{\partial c_{p}}{\partial \tilde{k}}\right)_{\tilde{\mu}}=2|\lambda| .
$$


Therefore, the group velocity exceeds unity on account of the condition (2.19). Because the boundary theory is non-gravitational, this is an unambiguous signal of causality violation.

This problem can be avoided by requiring that the conditions (2.18)-(2.19) be never satisfied. The only meaningful choice is to constrain the magnetic dipole coupling:

$$
\lambda^{2} \leq \frac{L^{2}}{4}
$$

where the AdS radius $L$ has been restored. Outside this range of values, $-\frac{L}{2} \leq \lambda \leq+\frac{L}{2}$, the boundary CFT will always be plagued with modes that propagate faster than light.

\subsection{WKB approximation: a numerical study}

In this section, we employ the WKB approximation method to reconfirm the occurrence of causality violation for $\lambda \notin\left[-\frac{L}{2},+\frac{L}{2}\right]$. In the limit (2.14), one can take $\hbar \equiv \frac{1}{\tilde{k}}$ and follow refs. [4-14] to write down a Bohr-Sommerfeld quantization condition, which enables one to compute the group velocity of the dual field theory modes.

Let us recall from eqs. (2.11)-(2.12) that while the chiral spinors $\psi_{ \pm}$are decoupled from each other, their components themselves are not. Then, we start with the WKB ansatz:

$$
\psi_{ \pm}^{\alpha}(\zeta)=\exp \left[i \tilde{k} S_{ \pm}^{\alpha}(\zeta)\right], \quad \alpha=1,2
$$

where $\alpha$ labels the components of $\psi_{ \pm}$, and $S_{ \pm}^{\alpha}$ has an expansion in negative powers of $\tilde{k}$. In order for the ansatz (2.28) to satisfy eq. (2.11) to all orders in $\tilde{k}$, it is required that $S_{ \pm}^{\alpha}$ contain half-integer powers of $\tilde{k}$ as well. Explicitly,

$$
S_{ \pm}^{\alpha} \equiv S_{0 \pm}^{\alpha}+\tilde{k}^{-1} S_{1 \pm}^{\alpha}+\tilde{k}^{-\frac{3}{2}} S_{\frac{3}{2} \pm}^{\alpha}+\tilde{k}^{-2} S_{2 \pm}^{\alpha}+\cdots
$$

Substituting eq. (2.28)-(2.29) into eq. (2.11) one finds that the components of each chiral spinor also get decoupled and obey identical equations to the leading order in $\frac{1}{\tilde{k}}$. As a result, $S_{0 \pm}^{\alpha}=S_{0 \pm}$, i.e., the leading-order WKB phase is independent of the channel. The WKB momentum, $p_{ \pm}(\zeta) \equiv S_{0 \pm}^{\prime}(\zeta)$, does not depend on the parameter $w$, and is given by

$$
p_{ \pm}^{2}(\zeta)=\frac{(1 \pm 2 v \zeta \lambda / L)^{2} f(\zeta)-(u+v-v \zeta)^{2}}{f^{2}(\zeta)}
$$

To proceed, an additional assumption is required, namely $S_{1 \pm}^{\alpha}=S_{1 \pm}$. Note that the imaginary part of $S_{1 \pm}$ gives the WKB amplitude, while the real part the first-order phase correction, and again these are channel independent. Now one can go on with the requirement that the determinant of the $2 \times 2$ coefficient matrix of $\psi_{ \pm}$vanish order by order in $\frac{1}{\tilde{k}}$ (see, for example, refs. [51, 52] for a discussion). At the next-to-leading order, the above requirement results in an algebraic equation that determines $S_{1 \pm}^{\prime}(\zeta)$, namely

$$
S_{1 \pm}^{\prime}(\zeta)=i \partial_{\zeta}\left(\ln \sqrt{p_{ \pm}(\zeta)}\right)+\text { Real Part }
$$

Clearly, the amplitude is inversely proportional to $\sqrt{p(\zeta)}$ like the single-channel case. 


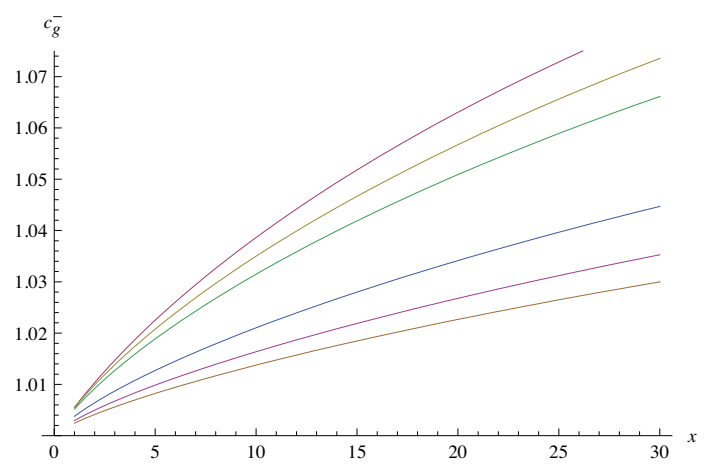

Figure 3. $\quad c_{g}^{-}$is plotted against $x \equiv\left(\frac{\lambda}{L}-\frac{1}{2}\right) \times$ $10^{3}$ for $(u, v)$ lying on the plane (2.18). Different colors correspond to different values of $v=2,3,5,10,15,20$. The group velocity is found to exceed unity.

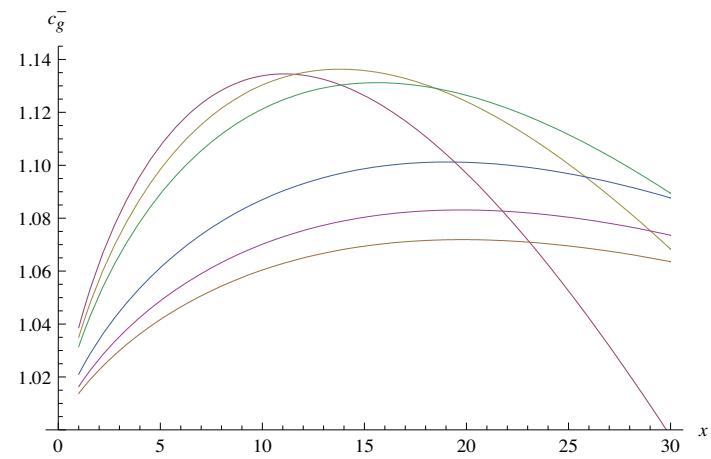

Figure 4. $\quad c_{g}^{-}$is plotted against $x \equiv\left(\frac{\lambda}{L}-\frac{1}{2}\right) \times$ $10^{2}$ for $(u, v)$ lying on the plane (2.18). Different colors correspond to different values of $v=2,3,5,10,15,20$. The group velocity is found to exceed unity.

Normalizability implies that $p_{ \pm}^{2}(\zeta)<0$ in the boundary neighborhood. In the bulk there may exist turning points $\zeta_{i}$, where the WKB momentum vanishes: $p_{ \pm}\left(\zeta_{i}\right)=0$. For two turning points $\zeta_{1}$ and $\zeta_{2}$, one is lead to the Bohr-Sommerfeld quantization condition:

$$
\tilde{k} \int_{\zeta_{1}}^{\zeta_{2}} d \zeta p_{ \pm}(\zeta)+\mathcal{O}\left(\tilde{k}^{0}\right)=\pi\left(n-\frac{1}{2}\right), \quad \zeta_{2}>\zeta_{1} \quad \text { and } \quad n=1,2, \ldots
$$

From the holographic point of view, this can be understood as a dispersion relation for the boundary modes coupled to the dual fermionic operator. Their group velocity $\left(\frac{\partial \tilde{\omega}}{\partial \tilde{k}}\right)_{\tilde{\mu}}$ can be computed by differentiating both sides of eq. (2.32). The result is

$$
c_{g}^{ \pm}=\frac{\int_{\zeta_{1}}^{\zeta_{2}} d \zeta\left(u \frac{\partial p_{ \pm}}{\partial u}+v \frac{\partial p_{ \pm}}{\partial v}-p_{ \pm}\right)}{\int_{\zeta_{1}}^{\zeta_{2}} d \zeta\left(\frac{\partial p_{ \pm}}{\partial u}\right)} .
$$

In what follows we resort to numerics to find some regions in the parameter space that allow for a pair of turning points to exist. This in turn enables one to evaluate numerically the group velocity from eq. (2.33). We take $\lambda>0$ and focus on the chiral spinor $\psi_{-}$that serves our purpose (for $\lambda<0$ we need to consider $\psi_{+}$instead). For simplicity, we further restrict the exploration on the plane defined by eq. (2.18) in the 3D space of $(u, v, \lambda)$. The existence of turning points crucially depends on the values of the parameters: at $\lambda=\frac{L}{2}$, a single turning point exists at $\zeta=\frac{1}{v}$. For $\lambda>\frac{L}{2}$, we distinguish two turning points $\zeta_{1}$ and $\zeta_{2}$, whose precise positions depend on the parameter values. For fixed $v$, the distance $\zeta_{2}-\zeta_{1}$ grows with $\lambda$ as $\zeta_{2}$ moves towards the horizon. For fixed $\lambda$ and increasing $v$, on the other hand, both turning points move closer to the boundary.

Figures 3 and 4 show that the group velocity $c_{g}^{-}$may indeed become superluminal for $\lambda>\frac{L}{2}$. For $\lambda$ slightly larger than this value, causality violation is observed numerically, on the plane (2.18) in the parameter space, for $v>1$. As $\lambda$ increases, acausality may only be captured by sufficiently large values of $v$. Note that the group velocity formula (2.33) 
is independent of the parameter $w$, and so one can choose the latter quantity large enough in order to accommodate large values of $v$ without violating the condition (2.15).

Thus sections 2.2 and 2.3 independently confirm our result (2.18). But they differ in methodology and the region of parameter space taken into account. In section 2.2 , we took $v \approx 1$ since otherwise the maximum (2.23) would not lie close to the boundary, and this would invalidate the whole argument (recall that the single-channel problem obtained in the limit (2.14) would cease to make sense in the near-horizon region). Moreover, the condition (2.18), necessary for the existence of a potential well near the boundary, was postulated to be the dispersion relation of the boundary modes. In contrast, in section 2.3 the parameter $v$ was free to take large values, and causality violation was seen only for $v>1$ on the plane (2.18). More importantly, the boundary dispersion relation (2.32) was actually derived rather than postulated, which makes the analysis more rigorous.

\section{Implications \& remarks}

We have shown that the AdS/CFT correspondence poses non-trivial constraints on the EM interactions of a charged massive Dirac particle: the otherwise undetermined strength of the dipole coupling of the classical bulk theory must have an upper bound. We reduced the problem to a zero-energy Schrödinger equation and argued about the existence of normalizable solutions peaked near the boundary when the parameters obey a certain relation. The latter served as a dispersion relation for some corresponding boundary modes, which are found to propagate superluminally when the above bound is violated. We also solved the coupled system of differential equations using the WKB approximation, and numerically confirmed that the group velocity exceeds unity above this bound.

The bulk dipole coupling changes the structure of current-fermion-fermion 3-point functions of the CFT, and one would like to investigate this point further. From the CFT point of view, constraints on $\lambda$ presumably correspond to constraints on the 4-point functions derived from unitarity and crossing symmetry. A promising direction could go along the lines of ref. [53], where constraints related to charged operators were obtained.

Obtained by considering a probe fermion in the AdS Reissner-Nordström geometry, our results are directly relevant for the physics of holographic non-Fermi liquids. Because large values of $\lambda^{2}$ are not physically meaningful, the disappearance of the Fermi surface and the onset of a gap, reported in ref. [27, 28] for $\lambda^{2} \simeq 4 L^{2}$, can actually never happen. Moreover, the shift in Fermi momentum observed in [23] for varying $\lambda$ can only be small. In other words, not only is the existence the Fermi surface robust but also the position thereof is sort of rigid for the allowed values of the dipole strength. This fact is also supported by the study of several top-down models, e.g. [46, 47].

A generic background may or may not have a holographic dual, but can only sharpen the bound (2.27). On the other hand, one might argue that a more extensive holographic analysis covering other regions of the parameter space could yield stronger constraints. In all the known examples [4, 5, 8-14], however, it has been only the high momentum limit that revealed the CFT pathology. This may not come as a surprise since causality is likely to be connected to the local, short-distance behavior of the theory $[4,5]$. 
We have seen that a bulk theory with no known classical inconsistency may have a boundary dual that exhibits pathologies. One is tempted to think that the CFT actually probes the quantum consistency of the theory in the bulk, and that the duality holds good only if the latter is well behaved quantum mechanically, at least in the weak coupling regime. An example that seems to justify this point is the existence of some classically consistent Vasiliev-like theories in $\mathrm{AdS}_{3}$ that are believed not to have healthy quantum versions since the dual CFTs are non-unitary [54].

\section{Acknowledgments}

We would like to thank X.O. Camanho, T.R. Govindarajan, Y. Korovin, A. Parnachev, A.O. Starinets and A. Zhiboedov for useful discussions. MK would like to thank the organizers of the "8th Crete Regional Meeting in String Theory," the "9th QTS Meeting in Yerevan" and the Galileo Galilei Institute for Theoretical Physics for the hospitality, and the INFN for partial support during the completion of this work. MK is supported in part by an NWO Vidi grant and by a Teaching Fellowship at Trinity College Dublin.

Open Access. This article is distributed under the terms of the Creative Commons Attribution License (CC-BY 4.0), which permits any use, distribution and reproduction in any medium, provided the original author(s) and source are credited.

\section{References}

[1] J.M. Maldacena, The large- $N$ limit of superconformal field theories and supergravity, Int. J. Theor. Phys. 38 (1999) 1113 [hep-th/9711200] [INSPIRE].

[2] S.S. Gubser, I.R. Klebanov and A.M. Polyakov, Gauge theory correlators from noncritical string theory, Phys. Lett. B 428 (1998) 105 [hep-th/9802109] [InSPIRE].

[3] E. Witten, Anti-de Sitter space and holography, Adv. Theor. Math. Phys. 2 (1998) 253 [hep-th/9802150] [INSPIRE].

[4] M. Brigante, H. Liu, R.C. Myers, S. Shenker and S. Yaida, The Viscosity Bound and Causality Violation, Phys. Rev. Lett. 100 (2008) 191601 [arXiv:0802.3318] [INSPIRE].

[5] M. Brigante, H. Liu, R.C. Myers, S. Shenker and S. Yaida, Viscosity Bound Violation in Higher Derivative Gravity, Phys. Rev. D 77 (2008) 126006 [arXiv:0712.0805] [INSPIRE].

[6] A. Buchel and R.C. Myers, Causality of Holographic Hydrodynamics, JHEP 08 (2009) 016 [arXiv: 0906.2922] [INSPIRE].

[7] D.M. Hofman, Higher Derivative Gravity, Causality and Positivity of Energy in a UV complete QFT, Nucl. Phys. B 823 (2009) 174 [arXiv:0907.1625] [InSPIRE].

[8] A. Buchel, J. Escobedo, R.C. Myers, M.F. Paulos, A. Sinha and M. Smolkin, Holographic GB gravity in arbitrary dimensions, JHEP 03 (2010) 111 [arXiv:0911.4257] [INSPIRE].

[9] J. de Boer, M. Kulaxizi and A. Parnachev, $A d S_{7} / C F T_{6}$, Gauss-Bonnet Gravity and Viscosity Bound, JHEP 03 (2010) 087 [arXiv:0910.5347] [INSPIRE].

[10] X.O. Camanho and J.D. Edelstein, Causality constraints in AdS/CFT from conformal collider physics and Gauss-Bonnet gravity, JHEP 04 (2010) 007 [arXiv:0911.3160] [INSPIRE]. 
[11] J. de Boer, M. Kulaxizi and A. Parnachev, Holographic Lovelock Gravities and Black Holes, JHEP 06 (2010) 008 [arXiv:0912.1877] [INSPIRE].

[12] X.O. Camanho and J.D. Edelstein, Causality in AdS/CFT and Lovelock theory, JHEP 06 (2010) 099 [arXiv: 0912.1944] [INSPIRE].

[13] X.O. Camanho, J.D. Edelstein and M.F. Paulos, Lovelock theories, holography and the fate of the viscosity bound, JHEP 05 (2011) 127 [arXiv: 1010.1682] [INSPIRE].

[14] R.C. Myers, M.F. Paulos and A. Sinha, Holographic studies of quasi-topological gravity, JHEP 08 (2010) 035 [arXiv: 1004.2055] [INSPIRE].

[15] D.M. Hofman and J. Maldacena, Conformal collider physics: Energy and charge correlations, JHEP 05 (2008) 012 [arXiv: 0803.1467] [INSPIRE].

[16] M. Kulaxizi and A. Parnachev, Energy Flux Positivity and Unitarity in CFTs, Phys. Rev. Lett. 106 (2011) 011601 [arXiv: 1007.0553] [INSPIRE].

[17] X.O. Camanho, J.D. Edelstein, J. Maldacena and A. Zhiboedov, Causality Constraints on Corrections to the Graviton Three-Point Coupling, arXiv: 1407.5597 [INSPIRE].

[18] H. Aronson, Spin-1 electrodynamics with an electric quadrupole moment, Phys. Rev. 186 (1969) 1434 [INSPIRE].

[19] G. Velo and D. Zwanziger, Noncausality and other defects of interaction lagrangians for particles with spin one and higher, Phys. Rev. 188 (1969) 2218 [INSPIRE].

[20] J.M. Cornwall, D.N. Levin and G. Tiktopoulos, Uniqueness of spontaneously broken gauge theories, Phys. Rev. Lett. 30 (1973) 1268 [Erratum ibid. 31 (1973) 572] [InSPIRE].

[21] J.M. Cornwall, D.N. Levin and G. Tiktopoulos, Derivation of Gauge Invariance from High-Energy Unitarity Bounds on the s Matrix, Phys. Rev. D 10 (1974) 1145 [Erratum ibid. D 11 (1975) 972] [INSPIRE].

[22] M. Kulaxizi and R. Rahman, Holographic Constraints on a Vector Boson, JHEP 04 (2013) 164 [arXiv: 1212.6265] [INSPIRE].

[23] D. Guarrera and J. McGreevy, Holographic Fermi surfaces and bulk dipole couplings, arXiv:1102.3908 [INSPIRE].

[24] S. Weinberg, The Quantum theory of fields. Vol. 1: Foundations, Cambridge University Press, Cambridge U.K. (1995), pg. 609.

[25] H. Liu, J. McGreevy and D. Vegh, Non-Fermi liquids from holography, Phys. Rev. D 83 (2011) 065029 [arXiv:0903.2477] [INSPIRE].

[26] T. Faulkner, H. Liu, J. McGreevy and D. Vegh, Emergent quantum criticality, Fermi surfaces and $A d S_{2}$, Phys. Rev. D 83 (2011) 125002 [arXiv:0907.2694] [inSPIRE].

[27] M. Edalati, R.G. Leigh and P.W. Phillips, Dynamically Generated Mott Gap from Holography, Phys. Rev. Lett. 106 (2011) 091602 [arXiv: 1010.3238] [INSPIRE].

[28] M. Edalati, R.G. Leigh, K.W. Lo and P.W. Phillips, Dynamical Gap and Cuprate-like Physics from Holography, Phys. Rev. D 83 (2011) 046012 [arXiv:1012.3751] [InSPIRE].

[29] W.-J. Li and H.-b. Zhang, Holographic non-relativistic fermionic fixed point and bulk dipole coupling, JHEP 11 (2011) 018 [arXiv:1110.4559] [INSPIRE].

[30] W.-J. Li, R. Meyer and H.-b. Zhang, Holographic non-relativistic fermionic fixed point by the charged dilatonic black hole, JHEP 01 (2012) 153 [arXiv:1111.3783] [INSPIRE]. 
[31] J.-P. Wu and H.-B. Zeng, Dynamic gap from holographic fermions in charged dilaton black branes, JHEP 04 (2012) 068 [arXiv: 1201.2485] [INSPIRE].

[32] W.-Y. Wen and S.-Y. Wu, Dipole Coupling Effect of Holographic Fermion in Charged Dilatonic Gravity, Phys. Lett. B 712 (2012) 266 [arXiv:1202.6539] [INSPIRE].

[33] X.-M. Kuang, B. Wang and J.-P. Wu, Dipole Coupling Effect of Holographic Fermion in the Background of Charged Gauss-Bonnet AdS Black Hole, JHEP 07 (2012) 125 [arXiv: 1205.6674] [INSPIRE].

[34] X.-M. Kuang, B. Wang and J.-P. Wu, Dynamical gap from holography in the charged dilaton black hole, Class. Quant. Grav. 30 (2013) 145011 [arXiv:1210.5735] [INSPIRE].

[35] L.Q. Fang, X.-H. Ge and X.-M. Kuang, Holographic fermions with running chemical potential and dipole coupling, Nucl. Phys. B 877 (2013) 807 [arXiv:1304.7431] [inSPIRE].

[36] Z. Fan, Dynamic Mott gap from holographic fermions in geometries with hyperscaling violation, JHEP 08 (2013) 119 [arXiv:1305.1151] [INSPIRE].

[37] G. Vanacore and P.W. Phillips, Minding the Gap in Holographic Models of Interacting Fermions, Phys. Rev. D 90 (2014) 044022 [arXiv: 1405.1041] [InSPIRE].

[38] J. Alsup, E. Papantonopoulos, G. Siopsis and K. Yeter, Duality between zeroes and poles in holographic systems with massless fermions and a dipole coupling, Phys. Rev. D 90 (2014) 126013 [arXiv: 1404.4010] [INSPIRE].

[39] M. Graña, D3-brane action in a supergravity background: The Fermionic story, Phys. Rev. D 66 (2002) 045014 [hep-th/0202118] [INSPIRE].

[40] M. Ammon, J. Erdmenger, M. Kaminski and A. O'Bannon, Fermionic Operator Mixing in Holographic p-wave Superfluids, JHEP 05 (2010) 053 [arXiv:1003.1134] [INSPIRE].

[41] I. Bah, A. Faraggi, J.I. Jottar, R.G. Leigh and L.A. Pando Zayas, Fermions and D $=11$ Supergravity On Squashed Sasaki-Einstein Manifolds, JHEP 02 (2011) 068 [arXiv: 1008.1423] [INSPIRE].

[42] I. Bah, A. Faraggi, J.I. Jottar and R.G. Leigh, Fermions and Type IIB Supergravity On Squashed Sasaki-Einstein Manifolds, JHEP 01 (2011) 100 [arXiv:1009.1615] [INSPIRE].

[43] O. DeWolfe, S.S. Gubser and C. Rosen, Fermi Surfaces in Maximal Gauged Supergravity, Phys. Rev. Lett. 108 (2012) 251601 [arXiv:1112.3036] [INSPIRE].

[44] O. DeWolfe, S.S. Gubser and C. Rosen, Fermi surfaces in $N=4$ super-Yang-Mills theory, Phys. Rev. D 86 (2012) 106002 [arXiv:1207.3352] [INSPIRE].

[45] O. DeWolfe, S.S. Gubser and C. Rosen, Fermionic response in a zero entropy state of $\mathcal{N}=4$ super-Yang-Mills, Phys. Rev. D 91 (2015) 046011 [arXiv:1312.7347] [InSPIRE].

[46] J.P. Gauntlett, J. Sonner and D. Waldram, Universal fermionic spectral functions from string theory, Phys. Rev. Lett. 107 (2011) 241601 [arXiv:1106.4694] [INSPIRE].

[47] J.P. Gauntlett, J. Sonner and D. Waldram, Spectral function of the supersymmetry current, JHEP 11 (2011) 153 [arXiv:1108.1205] [INSPIRE].

[48] L.J. Romans, Supersymmetric, cold and lukewarm black holes in cosmological Einstein-Maxwell theory, Nucl. Phys. B 383 (1992) 395 [hep-th/9203018] [INSPIRE].

[49] A. Chamblin, R. Emparan, C.V. Johnson and R.C. Myers, Charged AdS black holes and catastrophic holography, Phys. Rev. D 60 (1999) 064018 [hep-th/9902170] [INSPIRE]. 
[50] A. Chamblin, R. Emparan, C.V. Johnson and R.C. Myers, Holography, thermodynamics and fluctuations of charged AdS black holes, Phys. Rev. D 60 (1999) 104026 [hep-th/9904197] [INSPIRE].

[51] K. Yabana and H. Horiuchi, Relativistic Chiral Mean Field Model for Finite Nuclei, Prog. Theor. Phys. 71 (1984) 1275.

[52] Relativistic Chiral Mean Field Model for Finite Nuclei, Prog. Theor. Phys. 75 (1986) 592.

[53] Z. Komargodski and A. Zhiboedov, Convexity and Liberation at Large Spin, JHEP 11 (2013) 140 [arXiv: 1212.4103] [INSPIRE].

[54] E. Perlmutter, T. Prochazka and J. Raeymaekers, The semiclassical limit of $W_{N}$ CFTs and Vasiliev theory, JHEP 05 (2013) 007 [arXiv: 1210.8452] [INSPIRE]. 